\title{
Common fixed point theorems in non-Archimedean fuzzy metric-like spaces with applications
}

\author{
Haiqing Zhao a, Yanxia Lü ${ }^{a}$, Phikul Sridarat ${ }^{b}$, Suthep Suantai, ${ }^{b, *}$, Yeol Je Cho ${ }^{c, d, *}$ \\ a Department of Mathematics and Physics, Baoding 071003, China. \\ ${ }^{b}$ Department of Mathematics, Faculty of Science, Chiang Mai University, Chiang Mai, 50200, Thailand. \\ ${ }^{c}$ Department of Mathematics Education and RINS, Gyeongsang National University, Jinju 660-701, Korea. \\ ${ }^{d}$ Center for General Education, China Medical University, Taichung 40402, Taiwan.
}

Communicated by R. Saadati

\begin{abstract}
In this paper, we introduce the new concept called a non-Archimedean fuzzy metric-like space and prove some common fixed point theorems in this space. Our results extend some corresponding ones in the literature. Also, we give some examples to illustrate the main results. Finally, as applications, we consider the existence problem of solutions of integral equations by our main results. (C)2017 All rights reserved.
\end{abstract}

Keywords: Fuzzy metric space, metric-like space, Cauchy sequence, fixed point theorem.

2010 MSC: $47 \mathrm{H} 05,47 \mathrm{H} 09,54 \mathrm{H} 25$.

\section{Introduction}

In 1975, Kramosil and Michalek [12] introduced the concept called a fuzzy metric space. Later, some authors introduced some kinds of new fuzzy metric spaces in different ways $([4,5,11])$.

To obtain a Hausdorff topology of fuzzy metric spaces, in [7], George and Veeramani modified the concept of a fuzzy metric space in the sense of Kramosil and Michalek and showed that every ordinary metric induces a fuzzy metric in the sense of George and Veeramani.

On the other hand, in [8], Grabiec initiated the study of fixed point theory in fuzzy metric spaces, which is a parallel theory in probabilistic metric spaces [3]. In [9], Gregori and Sapena introduced a class of fuzzy contractive mappings and proved several fixed point theorems for these mappings in fuzzy metric spaces in the senses of George and Veeramani and Kramosil and Michalek. The results of Gregori and Sapena extend classical Banach's fixed point theorem, that is, their results can be considered a fuzzy version of Banach's contraction theorem. For more results on fixed point theorems in fuzzy metric spaces, the readers may refer to $[6,13-16,18,20,21]$.

\footnotetext{
${ }^{*}$ Corresponding author.

Email addresses: haiqingzhaoncepu@163.com (Haiqing Zhao), yanxialuncepu@163.com (Yanxia Lu), phikul_s@cmu.ac.th (Phikul Sridarat), suthep.s@cmu.ac.th (Suthep Suantai), yjcho@gnu.ac.kr (Yeol Je Cho)
} 
Recently, in [1, 2], Aydi et al. introduced the concept of a metric-like space and they proved some fixed point theorems in metric-like spaces. Based on the concept of a metric-like space, in [17], Hierro and Sen proposed a new space called a Menger probabilistic metric-like space, which is a generalization of a metric-like space, and proved some fixed point theorems in this space.

In this paper, inspired by the results of Hierro and Sen [17], we give the concept called a fuzzy metriclike space and point out the difference between these classes of fuzzy metric-like spaces and probabilistic metric-like spaces. Also, we give some examples to illustrate the class of fuzzy metric-like spaces. Then we define the concept of a non-Archimedean fuzzy metric-like space and prove some common fixed point theorems for some contractions in these spaces. Our results extend the corresponding ones in the literature and we give one example to show the validity of our main result. Finally, as applications, we consider the existence problem of solutions of integral equations by the results of this paper.

\section{Preliminaries}

First, we introduce some definitions and examples as follows:

Definition 2.1. A mapping $*:[0,1] \times[0,1] \rightarrow[0,1]$ is said to be a triangular norm (for short, a t-norm) if for all $a, b, c, d \in[0,1]$,

(t1) $1 * a=a * 1=a$;

(t2) $a * b \leqslant c * d$ if $a \leqslant c$ and $b \leqslant d ;$

(t3) $\mathrm{a} * \mathrm{~b}=\mathrm{b} * \mathrm{a}$;

(t4) $\mathrm{a} *(\mathrm{~b} * \mathrm{c})=(\mathrm{a} * \mathrm{~b}) * \mathrm{c}$.

It is known that $a *_{M} b=\min \{a, b\}$ and $a *_{p} b=a b$ are two classic continuous t-norms.

Definition 2.2 ([12]). A fuzzy metric space (in the sense of Kramosil and Michalek) is a triple $(X, M, *)$, where $X$ is a nonempty set, $*$ is a continuous t-norm and $M$ is a fuzzy set on $X^{2} \times[0, \infty)$ satisfying the following properties: for all $x, y, z \in X$ and $s, t>0$,

(KM1) $M(x, y, 0)=0$ for all $x, y \in X$;

(KM2) $M(x, y, t)=1$ for all $t>0$ if and only if $x=y$;

(KM3) $M(x, y, t)=M(y, x, t)$ for all $x, y \in X$ and $t>0$;

(KM4) $M(x, y, \cdot):[0, \infty) \rightarrow[0,1]$ is left continuous;

(KM5) $M(x, z, s+t) \geqslant M(x, y, s) * M(y, z, t)$.

From (KM2) and (KM5), we see that the fuzzy metric $M(x, y, t)$ is nondecreasing in $t$ for all $x, y \in X$ (see [8]).

In Definition 2.2, if (KM5) is replaced by

(NA) $M(x, z, \max \{s, t\}) \geqslant M(x, y, s) * M(y, z, t)$, for all $x, y, z \in X$ and $s, t,>0$

or, equivalently,

$$
M(x, z, t) \geqslant M(x, y, t) * M(y, z, t)
$$

for all $x, y, z \in X$ and $t>0$,

then the triple $(X, M, *)$ is called a non-Archimedean fuzzy metric space [10].

It is easy to see that (NA) implies (KM5), that is, each non-Archimedean fuzzy metric space itself is a fuzzy metric space. However, the inverse is not true.

In this paper, for our purpose, we give the new concept called a fuzzy metric-like space (in the sense of Kramosil and Michalek) by modifying Definition 2.2. 
Definition 2.3. Let $X$ be a nonempty set, $*$ be a continuous t-norm and $M$ be a fuzzy on $X^{2} \times[0, \infty)$. A triple $(X, M, *)$ is called a fuzzy metric-like space, if the following hold: for all $x, y, z \in X$ and $s, t>0$,

(KML1) $M(x, y, 0)=0$;

(KML2) $M(x, y, t)=1$ implies $x=y$;

(KML3) $M(x, y, t)=M(y, x, t)$;

(KML4) $M(x, y, \cdot):[0, \infty) \rightarrow[0,1]$ is left continuous;

(KML5) $M(x, z, s+t) \geqslant M(x, y, s) * M(y, z, t)$.

Example 2.4. Let $X=[0, \infty)$ and $M: X^{2} \times[0, \infty) \rightarrow[0,1]$ be defined by $M(x, y, 0)=0$ for all $x, y \in X$ and

$$
M(x, y, t)=\frac{t}{t+x+y}
$$

for all $x, y \in X$ and $t>0$. It is easy to see that $M$ satisfies the conditions (KML1)-(KML4).

Now, we show that

$$
\frac{s+t}{s+t+x+z} \geqslant \min \left\{\frac{s}{s+x+y}, \frac{t}{t+y+z}\right\}
$$

for all $x, y, z \in X$ and $s, t>0$. Without loss of generality, we assume that $\frac{s}{s+x+y} \geqslant \frac{t}{t+y+z}$, i.e., $s(y+z) \geqslant t(x+y)$. It follows that

$$
t(y+z)+s(y+z) \geqslant t(y+z+x+y),
$$

which implies that

$$
\frac{s+t}{s+t+x+y+y+z} \geqslant \frac{t}{t+y+z} .
$$

Since $\frac{s+t}{s+t+x+y+y+z} \leqslant \frac{s+t}{s+t+x+z}$, we have

$$
\frac{t}{t+y+z} \leqslant \frac{s+t}{s+t+x+z}
$$

Thus (2.1) holds. Therefore, $\left(X, M, *_{M}\right)$ is a fuzzy metric-like space. It is easy to see that $\left(X, M, *_{p}\right)$ is also a fuzzy metric-like space since $*_{M} \geqslant * p$.

Example 2.5. Let $X=(-\infty, \infty)$ and $M: X^{2} \times[0, \infty) \rightarrow[0,1]$ be defined by $M(x, y, 0)=0$ for all $x, y \in X$ and

$$
M(x, y, t)=e^{-\frac{x^{2}+y^{2}}{t}}
$$

for all $x, y \in X$ and $t>0$. If $M(x, y, t)=1$ for all $t>0$, then $x=y=0$. Hence (KML1) holds. Obviously, (KML2)-(KML4) also hold.

Now, we show that

$$
e^{-\frac{x^{2}+z^{2}}{s+t}} \geqslant e^{-\frac{x^{2}+y^{2}}{s}} e^{-\frac{y^{2}+z^{2}}{t}}
$$

for all $x, y, z \in X$ and $s, t>0$. In fact, from

$$
x^{2}+z^{2} \leqslant \frac{s+t}{t}\left(x^{2}+y^{2}\right)+\frac{s+t}{s}\left(y^{2}+z^{2}\right),
$$

we have

$$
\frac{x^{2}+z^{2}}{s+t} \leqslant \frac{x^{2}+y^{2}}{s}+\frac{y^{2}+z^{2}}{t}
$$

and so

$$
e^{\frac{x^{2}+z^{2}}{s+t}} \leqslant e^{\frac{x^{2}+y^{2}}{s}} e^{\frac{y^{2}+z^{2}}{t}},
$$

which implies that (2.2) holds. Therefore, $\left(X, M, *_{p}\right)$ is a fuzzy metric-like space. 
In Example 2.4, the function $M(x, y, t)$ is non-decreasing in $t$ for all $x, y \in X$. However, in the following example, it is not this case.

Example 2.6. Let $X=[0,1]$ and $M: X^{2} \times[0, \infty) \rightarrow[0,1]$ be defined by $M(x, y, 0)=0$ for all $x, y \in X$, $M(0,0, t)=1$ for all $t>0$ and $M(x, y, t)=\frac{x y}{e^{t}}$ for all $x, y \in X$ with $x \neq 0, y \neq 0$ and $t>0$. It is easy to check that $\left(X, M, *_{P}\right)$ is a fuzzy metric-like space. Moreover, for all $x, y \in X, M(x, y, t)$ is non-increasing in $t>0$.

From Example 2.6, we see the following:

Remark 2.7. In a fuzzy metric-like space $(X, M, *)$,

(1) even if $x=y$, then $M(x, y, t)=1$ for all $t>0$ does not necessarily hold. However, for some $x, y \in X$, $x=y$ may imply that $M(x, y, t)=1$ for all $t>0$. For example, in Example 2.6, $M(0,0, t)=1$ for all $\mathrm{t}>0$;

(2) for all $x, y \in X, M(x, y, t)$ is not necessary non-decreasing in $t>0$, which is the main difference with Menger probabilistic metric-like space defined by Hierro and Sen [17].

Let $(X, M, *)$ be a fuzzy metric-like space. For all $x \in X, \epsilon \in(0,1)$ and $t>0$, define

$$
B_{x}(\epsilon, t)=\{y \in X: M(x, y, t)>1-\epsilon\} .
$$

$B_{x}(\epsilon, t)$ is said to be an $(\epsilon, t)$-neighborhood field of $x$. It needs to notice that $x \in B_{x}(\epsilon, t)$ does not necessarily hold.

Definition 2.8. Let $(X, M, *)$ be a fuzzy metric-like space and $\left\{x_{n}\right\}$ be a sequence in $X$.

(1) $\left\{x_{n}\right\}$ is said to be convergent to $x \in X$ if for any $\epsilon \in(0,1)$ and $t>0$, there exists $N=N(\epsilon, t) \in \mathbb{N}$ such that $x_{n} \in B_{x}(\epsilon, t)$ for all $n>N$, that is, $\lim _{n \rightarrow \infty} M\left(x_{n}, x, t\right)=1$ for all $t>0$. We call the point $x$ as the limit of $\left\{x_{n}\right\}$ and write $x_{n} \rightarrow x$ as $n \rightarrow \infty$.

(2) $\left\{x_{n}\right\}$ is called a Cauchy sequence in $X$ if for any $\epsilon \in(0,1)$ and $t>0$, there exists $N=N(\epsilon, t) \in \mathbb{N}$ such that $x_{n} \in B_{x_{m}}(\epsilon, t)$ for all $m>n>N$, that is, for all $m>n>N, M\left(x_{n}, x_{m}, t\right)>1-\epsilon$.

(3) $(X, M, *)$ is said to be complete if each Cauchy sequence in $(X, M, *)$ is convergent to a point in $X$.

Proposition 2.9. Let $(X, M, *)$ be a fuzzy metric-like space and $\left\{x_{n}\right\}$ be a sequence in $X$. If $\left\{x_{n}\right\}$ is convergent, then the limit of $\left\{x_{n}\right\}$ is unique.

Proof. Assume that $x, y \in X$ are the limits of $\left\{x_{n}\right\}$. Now, we show that $x=y$. In fact, since $*$ is continuous, it follows from (KML5) that, for all $t>0$,

$$
M(x, y, t) \geqslant M\left(x, x_{n}, \frac{t}{2}\right) * M\left(x_{n}, y, \frac{t}{2}\right) \rightarrow 1 * 1=1
$$

as $n \rightarrow \infty$. So, it follows from (KML2) that $x=y$. This completes the proof.

In a fuzzy metric space $(X, M, *)$, if a sequence $\left\{x_{n}\right\}$ converges to a point $x \in X$, then $M\left(x_{n}, y, t\right)$ converges to $M(x, y, t)$ for all $y \in X$ and $t>0$. However, in a fuzzy metric-like space $(X, M, *)$, if a sequence $\left\{x_{n}\right\}$ in $X$ converges to a point $x \in X$, then it does not imply that $M\left(x_{n}, y, t\right) \rightarrow M(x, y, t)$ for all $y \in X$ and $t>0$. For this, we give the following example.

Example 2.10. Let $X=[1, \infty)$ and $M: X^{2} \times[0, \infty) \rightarrow[0,1]$ be defined by $M(x, y, 0)=0$ for all $x, y \in X$, $M(1,1, t)=1, M(1,4, t)=M(4,1, t)=\frac{1}{2}$ for all $t>0$ and $M(x, y, t)=\frac{1}{x y}$ for all $x, y \in X$ with $(x, y) \notin$ $\{(1,1),(1,4),(4,1)\}$ and $t>0$. It is easy to check that $\left(X, M, *_{P}\right)$ is a fuzzy metric-like space. Let $\left\{x_{n}\right\}$ be a sequence defined by $x_{n}=1+\frac{1}{n}$ for each $n \in \mathbb{N}$. Then

$$
M\left(x_{n}, 1, t\right)=M\left(1+\frac{1}{n}, 1, t\right)=\frac{1}{\left(1+\frac{1}{n}\right)} \rightarrow 1
$$

for all $t>0$ as $n \rightarrow \infty$, that is, $x_{n} \rightarrow 1$ as $n \rightarrow \infty$. However, it follows that 


$$
M(1,4, t)=\frac{1}{2}, \quad M\left(x_{n}, 4, t\right)=\frac{1}{4\left(1+\frac{1}{n}\right)} \rightarrow \frac{1}{4}
$$

for all $t>0$ as $n \rightarrow \infty$, that is, $M\left(x_{n}, 4, t\right) \nrightarrow M(1,4, t)$ for each $t>0$ as $n \rightarrow \infty$.

Non-Archimedean fuzzy metric-like space: In Definition 2.3, if we replace the condition (KML5) with the condition (NA), then we call $(X, M, *)$ a non-Archimedean fuzzy metric-like space. Of course, if $(X, M, *)$ is a non-Archimedean fuzzy metric-like space, then the following holds:

$$
M(x, z, t) \geqslant M(x, y, t) * M(y, z, t)
$$

for all $x, y, z \in X$ and $t>0$.

Now, we give one example which is a non-Archimedean fuzzy metric-like space, but not a fuzzy metric-like space since as follows:

Example 2.11. Let $X=\mathbb{N}$. Define a fuzzy set $M$ on $X^{2} \times[0, \infty)$ by $M(x, y, 0)=0$ for all $x, y \in X$, $M(1,1, t)=1$ for all $t>0$ and

$$
M(x, y, t)= \begin{cases}\frac{1}{5}, & \text { if } 0<t \leqslant \frac{1}{2} \\ \frac{3}{10}, & \text { if } \frac{1}{2}<t \leqslant 1 \\ \frac{1}{10}, & \text { if } t \geqslant 1 .\end{cases}
$$

It is easy to check that $\left(X, M, *_{M}\right)$ is a non-Archimedean fuzzy metric-like space. However, $\left(X, M, *_{M}\right)$ is not a fuzzy metric-like space since

$$
M\left(x, z, \frac{9}{10}+\frac{7}{10}\right)<\min \left\{M\left(x, y, \frac{9}{10}\right), M\left(y, z, \frac{7}{10}\right)\right\}
$$

for all $x, y, z \in X$.

Proposition 2.12. Let $(X, M, *)$ be a non-Archimedean fuzzy metric-like space and $\left\{x_{n}\right\},\left\{y_{n}\right\}$ be the sequences in $X$. Assume that $x_{n} \rightarrow x$ and $y_{n} \rightarrow y$ as $n \rightarrow \infty$. Then $\lim _{n \rightarrow \infty} M\left(x_{n}, y_{n}, t\right)=M(x, y, t)$ for all $t>0$.

Proof. For all $t>0$, we have

$$
M(x, y, t) \geqslant M\left(y, y_{n}, t\right) * M\left(y_{n}, x_{n}, t\right) * M\left(x_{n}, x, t\right)
$$

and

$$
M\left(x_{n}, y_{n}, t\right) \geqslant M\left(x_{n}, x, t\right) * M(x, y, t) * M\left(y, y_{n}, t\right)
$$

Since $*$ is continuous, we have

$$
M(x, y, t) \geqslant \limsup _{n \rightarrow \infty} M\left(y, y_{n}, t\right) * M\left(y_{n}, x_{n}, t\right) * M\left(x_{n}, x, t\right)=\limsup _{n \rightarrow \infty} M\left(y_{n}, x_{n}, t\right)
$$

and

$$
\liminf _{n \rightarrow \infty} M\left(x_{n}, y_{n}, t\right) \geqslant \liminf _{n \rightarrow \infty} M\left(x_{n}, x, t\right) * M(x, y, t) * M\left(y, y_{n}, t\right)=M(x, y, t) .
$$

Thus it follows that

$$
\lim _{n \rightarrow \infty} M\left(x_{n}, y_{n}, t\right)=M(x, y, t)
$$

This completes the proof.

\section{The main results}

Let $\Phi$ denote the set of all functions $\varphi:[0,1] \rightarrow[0, \infty)$ satisfying the following conditions:

(a) $\varphi(t)$ is strictly decreasing for all $t>0$ and left continuous;

(b) $\varphi^{-1}(0)=\{1\}$.

It is easy to see that, if $\varphi \in \Phi$, then $\varphi\left(a_{n}\right) \rightarrow 0$ if and only if $a_{n} \rightarrow 1$. 
Theorem 3.1. Let $(\mathrm{X}, \mathrm{M}, *)$ be a complete non-Archimedean fuzzy metric-like space and $\mathrm{S}, \mathrm{T}: \mathrm{X} \rightarrow \mathrm{X}$ be two mappings. Suppose that there exists $\varphi \in \Phi$ such that

$$
\varphi(M(S x, T y, t)) \leqslant k(t) \varphi(M(x, y, t))
$$

for all $\mathrm{x}, \mathrm{y} \in \mathrm{X}$ and $\mathrm{t}>0$, where $\mathrm{k}(\mathrm{t}) \in(0,1)$ for all $\mathrm{t}>0$. Then $\mathrm{S}$ and $\mathrm{T}$ have a unique common fixed point in $\mathrm{X}$. Proof. Take $x_{0} \in X$ arbitrarily and define the sequence $\left\{x_{n}\right\}$ in $X$ by

$$
\left\{\begin{array}{l}
x_{2 n+1}=S x_{2 n}, \\
x_{2 n+2}=T x_{2 n+1}
\end{array}\right.
$$

for each $n \in \mathbb{N} \cup\{0\}$. For each $n \in \mathbb{N} \cup\{0\}$, by (3.1) we have

$$
\varphi\left(M\left(x_{2 n+1}, x_{2 n+2}, t\right)\right)=\varphi\left(M\left(S x_{2 n}, T x_{2 n+1}, t\right)\right) \leqslant k(t) \varphi\left(M\left(x_{2 n}, x_{2 n+1}, t\right)\right)
$$

and

$$
\varphi\left(M\left(x_{2 n+3}, x_{2 n+2}, t\right)\right)=\varphi\left(M\left(S x_{2 n+2}, T x_{2 n+1}, t\right)\right) \leqslant k(t) \varphi\left(M\left(x_{2 n+2}, x_{2 n+1}, t\right)\right)
$$

for all $t>0$. It follows that, for each $n \in \mathbb{N}$,

$$
\varphi\left(M\left(x_{n}, x_{n+1}, t\right)\right) \leqslant k(t) \varphi\left(M\left(x_{n-1}, x_{n}, t\right)<\varphi\left(M\left(x_{n-1}, x_{n}, t\right)\right)\right.
$$

for all $t>0$. Since $\varphi$ is strictly decreasing, one has

$$
M\left(x_{n}, x_{n+1}, t\right)>M\left(x_{n-1}, x_{n}, t\right)
$$

for each $n \in \mathbb{N}$ and $t>0$. Let $t>0$ and $a_{n}(t)=M\left(x_{n}, x_{n+1}, t\right)$. Then there exists $a(t) \in(0,1]$ such that

$$
\lim _{n \rightarrow \infty} a_{n}(t)=a(t) \text {. }
$$

Since $\varphi$ is left continuous, it follows from (3.2) that

$$
\varphi(a(t)) \leqslant k(t) \varphi(a(t))<\varphi(a(t)),
$$

which is a contradiction. So, $\varphi(a(t))=0$ and $a(t)=1$. Thus we have

$$
\lim _{n \rightarrow \infty} M\left(x_{n}, x_{n+1}, t\right)=1
$$

for all $t>0$.

Next, we show that $\left\{x_{n}\right\}$ is a Cauchy sequence in $X$. In fact, if $\left\{x_{n}\right\}$ is not a Cauchy sequence, then there exist $\epsilon_{0} \in(0,1)$ and $t_{0}>0$ such that, for each $n \in \mathbb{N}$, there exist $p_{n}, q_{n} \in \mathbb{N}$ with $p_{n}>q_{n} \geqslant n$ satisfying

$$
M\left(x_{p_{n}}, x_{q_{n}}, t_{0}\right) \leqslant 1-\epsilon_{0}, \quad M\left(x_{p_{n}-1}, x_{q_{n}-1}, t_{0}\right)>1-\epsilon_{0} .
$$

By (3.3) and (note that (NA) implies that the following second inequality holds)

$$
1-\epsilon_{0} \geqslant M\left(x_{p_{n}}, x_{q_{n}}, t_{0}\right) \geqslant M\left(x_{p_{n}}, x_{q_{n}+1}, t_{0}\right) * M\left(x_{q_{n}+1}, x_{q_{n}}, t_{0}\right),
$$

we have

$$
\begin{aligned}
1-\epsilon_{0} & \geqslant \limsup _{n \rightarrow \infty} M\left(x_{\mathfrak{p}_{n}}, x_{\mathbf{q}_{n}+1}, t_{0}\right) * M\left(x_{\mathbf{q}_{n}+1}, x_{\mathbf{q}_{n}}, t_{0}\right) \\
& =\limsup _{n \rightarrow \infty} M\left(x_{\mathfrak{p}_{n}}, x_{\mathbf{q}_{n}+1}, t_{0}\right) .
\end{aligned}
$$

Similarly, we also have

$$
1-\epsilon_{0} \geqslant \limsup _{n \rightarrow \infty} M\left(x_{p_{n}+1}, x_{q_{n}}, t_{0}\right), \quad 1-\epsilon_{0} \geqslant \limsup _{n \rightarrow \infty} M\left(x_{p_{n}+1}, x_{q_{n}+1}, t_{0}\right) .
$$


Thus we can assume that $p_{n}$ are odd numbers, $q_{n}$ are even numbers and

$$
M\left(x_{p_{n}}, x_{q_{n}}, t_{0}\right) \leqslant 1-\epsilon_{0}
$$

for each $n \in \mathbb{N}$. By (3.1), we have

$$
\begin{aligned}
\varphi\left(M\left(x_{\mathfrak{p}_{\mathfrak{n}}}, x_{\mathbf{q}_{\mathfrak{n}}}, \mathrm{t}_{0}\right)\right) & =\varphi\left(M\left(S x_{\mathfrak{p}_{\mathfrak{n}}-1}, T x_{\mathbf{q}_{\mathfrak{n}}-1}, \mathrm{t}_{0}\right)\right) \\
& \leqslant k\left(\mathrm{t}_{0}\right) \varphi\left(M\left(x_{\mathfrak{p}_{\mathfrak{n}}-1}, x_{\mathbf{q}_{\mathfrak{n}}-1}, \mathrm{t}_{0}\right)\right) \\
& <\varphi\left(M\left(x_{\mathfrak{p}_{\mathfrak{n}}-1}, x_{\mathbf{q}_{\mathfrak{n}}-1}, \mathrm{t}_{0}\right)\right) .
\end{aligned}
$$

Since $\varphi$ is strictly decreasing, one has

$$
1-\epsilon_{0} \geqslant M\left(x_{p_{n}}, x_{q_{n}}, t_{0}\right)>M\left(x_{p_{n}-1}, x_{q_{n}-1}, t_{0}\right) \geqslant 1-\epsilon_{0},
$$

which is a contradiction. Thus $\left\{x_{n}\right\}$ is a Cauchy sequence in $X$. Since $X$ is complete, there exists a point $x \in X$ such that $\left\{x_{n}\right\}$ converges to $x$.

Now, we prove that the limit $x$ is a common fixed point of $S$ and $T$. For each $n \in \mathbb{N}$ and $t>0$, it follows from (3.1) that

$$
\begin{aligned}
\varphi\left(M\left(S x, x_{2 n+2}, t\right)\right) & =\varphi\left(M\left(S x, T x_{2 n+1}, t\right)\right) \\
& \leqslant k(t) \varphi\left(M\left(x, x_{2 n+1}, t\right)\right) \\
& \rightarrow k(t) \varphi(1)=0
\end{aligned}
$$

as $n \rightarrow \infty$. Since $\varphi$ is strictly decreasing and left continuous, we have

$$
\lim _{n \rightarrow \infty} M\left(S x, x_{2 n+2}, t\right)=1
$$

for all $t>0$. It follows that

$$
M(S x, x, t) \geqslant M\left(S x, x_{2 n+2}, t\right) * M\left(x_{2 n+2}, x, t\right) \rightarrow 1
$$

for all $t>0$ as $n \rightarrow \infty$. Thus $x=S x$. Similarly, we can have $x=T x$. Therefore, the limit $x$ is a common fixed point of $S$ and $T$.

Finally, we show the uniqueness of a common fixed point of the mappings $S$ and $T$. Suppose that $y(\neq x)$ is another common fixed point of $S$ and $T$. By (3.1), we have

$$
\varphi(M(x, y, t))=\varphi(M(S x, T y, t)) \leqslant k(t) \varphi(M(x, y, t))<\varphi(M(x, y, t))
$$

for all $t>0$. Hence $\varphi(M(x, y, t))=0$, which implies $M(x, y, t)=1$ for all $t>0$. Thus, from (KML2), it follows that $x=y$. This completes the proof.

Corollary 3.2. Let $(\mathrm{X}, \mathrm{M}, *)$ be a complete non-Archimedean fuzzy metric-like space and $\mathrm{S}: \mathrm{X} \rightarrow \mathrm{X}$ be a mapping. Suppose that there exists $\varphi \in \Phi$ such that

$$
\varphi(M(S x, S y, t)) \leqslant k(t) \varphi(M(x, y, t))
$$

for all $\mathrm{x}, \mathrm{y} \in \mathrm{X}$ and $\mathrm{t}>0$, where $\mathrm{k}(\mathrm{t}) \in(0,1)$ for all $\mathrm{t}>0$. Then $\mathrm{S}$ has a unique fixed point in $\mathrm{X}$.

Since every fuzzy metric-like space is not necessarily a non-Archimedean fuzzy metric-like space, we can not get the following result by directly replacing a non-Archimedean fuzzy metric-like space with a fuzzy metric-like space.

Theorem 3.3. Let $(X, M, *)$ be a complete fuzzy metric-like space and $S: X \rightarrow X$ be a mapping. Suppose that there exists $\varphi \in \Phi$ such that

$$
\varphi(M(S x, S y, t)) \leqslant k(t) \varphi(M(x, y, t)),
$$

for all $\mathrm{x}, \mathrm{y} \in \mathrm{X}$ and $\mathrm{t}>0$, where $\mathrm{k}(\mathrm{t}) \in(0,1)$ for all $\mathrm{t}>0$. Then $\mathrm{S}$ has a unique fixed point in $\mathrm{X}$. 
Proof. For each $n \in \mathbb{N}$, let $b_{n}(t)=\sup _{k \geqslant 1} \varphi\left(M\left(x_{n}, x_{n+k}, t\right)\right)$. From (3.4), it follows that, for each $n, k \in \mathbb{N}$,

$$
\begin{aligned}
\varphi\left(M\left(x_{n}, x_{n+k}\right), t\right) & =\varphi\left(M\left(S^{n} x_{0}, S^{n+k} x_{0}, t\right)\right) \\
& \leqslant k(t) \varphi\left(M\left(S^{n-1} x_{0}, S x^{n-1+k} x_{0}, t\right)\right) \\
& =k(t) \varphi\left(M\left(x_{n-1}, x_{n-1+k}, t\right)\right),
\end{aligned}
$$

which implies that

$$
b_{n}(t) \leqslant k(t) b_{n-1}(t)<b_{n-1}(t)
$$

for each $n \in \mathbb{N}$ and $t>0$. Thus there exists $b \in[0,1]$ such that $b_{n}(t) \rightarrow b(t)$ as $n \rightarrow \infty$. Further, we have $b(t) \leqslant k(t) b(t)<b(t)$. Hence $b(t)=0$ for all $t>0$. Noting that $\varphi\left(M\left(x_{n}, x_{n+k}, t\right)\right) \leqslant b_{n}$ for each $n, k \in \mathbb{N}$, we have

$$
\lim _{n \rightarrow \infty} \varphi\left(M\left(x_{n}, x_{n+k}, t\right)\right)=0
$$

for each $k \in \mathbb{N}$. Since $\varphi \in \Phi$, we can obtain

$$
\lim _{n \rightarrow \infty} M\left(x_{n}, x_{n+k}, t\right)=1
$$

for each $k \in \mathbb{N}$. It follows that $\left\{x_{n}\right\}$ is a Cauchy sequence in $X$. Since $X$ is complete, there exists $x^{*} \in X$ such that $x_{n} \rightarrow x^{*}$ as $n \rightarrow \infty$.

Finally, by the similar process with the proof of Theorem 3.1, we can conclude that $x^{*}$ is the unique fixed point of $S$. This completes the proof.

Remark 3.4. All the results above still hold if we replace a (non-Archimedean) fuzzy metric-like space with a (non-Archimedean) fuzzy metric space. Theorem 3.3 improves the result of Shen et al. [19] from a fuzzy metric space to a fuzzy metric-like space.

Now, we give an example to illustrate Theorem 3.1.

Example 3.5. Let $X=\left\{\frac{1}{n}: n \in \mathbb{N}\right\} \cup\{0\}$ and $M: X^{2} \times[0, \infty) \rightarrow[0,1]$ be defined by $M(x, y, 0)=0$ for all $x, y \in X, M(0,0, t)=1$ for all $t>0$ and $M(x, y, t)=e^{-\frac{x+y}{t}}$ for all $x, y \in X$ with $x+y>0$ and $t>0$.

Now, we show that $\left(X, M, *_{P}\right)$ is a non-Archimedean fuzzy metric-like space. First, it is easy to see that $M$ satisfies the conditions (KML1)-(KML4). For all $s, t>0$ and $x, y, z \in X$, since

$$
-(x+z) \leqslant \frac{-\max \{s, t\}(x+y)}{s}+\frac{-\max \{s, t\}(y+z)}{t},
$$

we have

$$
e^{-\frac{x+z}{\max \{s, t\}}} \geqslant e^{-\frac{x+y}{s}} e^{-\frac{y+z}{t}}
$$

that is,

$$
M(x, z, \max \{s, t\}) \geqslant M(x, y, s) *_{p} M(y, z, t)
$$

for all $x, y, z \in X$ and $s, t>0$. Therefore, $\left(X, M, *_{P}\right)$ is a non-Archimedean fuzzy metric-like space. Let $\left\{x_{n}\right\}$ be a Cauchy sequence in $X$. By the definition of $M$, there exists $N \in \mathbb{N}$ such that, for all $n>N$, $x_{n}=0$. Thus $(X, M, * p)$ is complete.

Let $\varphi(t)=-\ln t$ for all $t \in(0,1]$. Define two mappings $S, T: X \rightarrow X$ by $S(0)=0, S\left(\frac{1}{n}\right)=\frac{1}{2 n}, T(0)=0$ and $T\left(\frac{1}{n}\right)=\frac{1}{3 n}$ for each $n \in \mathbb{N}$. Let $k(t)=\frac{1}{2}$ for all $t>0$. Then we show that the mappings $S$ and $T$ satisfy

$$
\varphi(M(S x, T y, t)) \leqslant k(t) \varphi(M(x, y, t))
$$

for all $x, y \in X$ and $t>0$. It is obvious that (3.5) holds if $x=y=0$. For $x=0$ and $y=\frac{1}{n}$, we have

$$
\varphi(M(S x, T y, t))=\frac{1}{3 n t} \leqslant \frac{1}{2} \varphi(M(x, y, t))=\frac{1}{2 n t}
$$


for all $t>0$. Hence (3.5) holds. Similarly, (3.5) also holds if $x=\frac{1}{n}$ and $y=0$.

For $x=\frac{1}{n}$ and $y=\frac{1}{m}$, we have

$$
\varphi(M(S x, T y, t))=\frac{1}{(2 n+3 m) t} \leqslant \frac{1}{2} \varphi(M(x, y, t))=\frac{1}{(2 n+2 m) t}
$$

for all $t>0$. Hence (3.5) holds. Therefore, all the conditions in Theorem 3.1 are satisfied and so we can conclude that $S$ and $T$ have a unique common fixed point $x^{*}$ in $X$. In fact, $x^{*}=0$ is a unique common fixed point of the mappings $S$ and $T$.

\section{Applications}

In this section, consider the following integral equation:

$$
x(t)=\int_{0}^{L} K(t, r, x(r)) d r,
$$

where $\mathrm{L}>0$ and $\mathrm{K}:[0, \mathrm{~L}] \times[0, \mathrm{~L}] \times \mathbb{R} \rightarrow \mathbb{R}$ is a mapping.

Let $\mathrm{X}=\mathrm{C}[0, \mathrm{~L}]$ be the set of all continuous real functions on $[0, \mathrm{~L}]$ and define a mapping $\mathrm{M}: \mathrm{X} \times \mathrm{X} \times$ $[0, \infty) \rightarrow[0,1]$ by $M(u, v, 0)=0$ for all $u, v \in X$ and

$$
M(u, v, t)=e^{-\frac{\max _{x \in[0, \mathrm{~L}]}(|\mathbf{u}(\mathrm{x})|+|v(x)|)}{\mathrm{t}}}
$$

for all $u, v \in X$ and $t>0$. Now, we show that $\left(X, F, \Delta_{P}\right)$ is a fuzzy metric-like space. Obviously, $F$ satisfies the conditions (KML1)-(KML3). Next, we prove the following holds:

$$
M(u, v, s+t) \geqslant M(u, w, s) M(w, v, t)
$$

for all $u, v, w \in X$ and $s, t>0$. Observe that

$$
\max _{x \in[0, \mathrm{~L}]}(|\mathrm{u}(\mathrm{x})|+|v(x)|) \leqslant \frac{\mathrm{s}+\mathrm{t}}{\mathrm{s}} \max _{x \in[0, \mathrm{~L}]}(|\mathrm{u}(\mathrm{x})|+|w(x)|)+\frac{\mathrm{s}+\mathrm{t}}{\mathrm{t}} \max _{x \in[0, \mathrm{~L}]}(|w(x)|+|v(x)|) .
$$

So, it follows that

$$
\frac{1}{s+t} \max _{x \in[0, \mathrm{~L}]}(|u(x)|+|v(x)|) \leqslant \frac{1}{s} \max _{x \in[0, \mathrm{~L}]}(|u(x)|+|w(x)|)+\frac{1}{\mathrm{t}} \max _{x \in[0, \mathrm{~L}]}(|w(x)|+|v(x)|) .
$$

Hence we have

$$
\begin{aligned}
e^{-\frac{\max _{x \in[0, \mathrm{~L}]}(|\mathrm{u}(\mathrm{x})|+|v(x)|)}{s+\mathrm{t}}} & \geqslant e^{-\frac{\max _{x \in[0, \mathrm{~L}]}(|\mathbf{u}(\mathrm{x})|+|w(x)|)}{s}}-\frac{\max _{x \in[0, \mathrm{~L}]}(|w(x)|+|v(x)|)}{\mathrm{t}} \\
& =e^{-\frac{\max _{x \in[0, \mathrm{~L}]}(|\mathbf{u}(\mathrm{x})|+|w(x)|)}{\mathrm{s}}} e^{-\frac{\max _{x \in[0, \mathrm{~L}]}(|w(x)|+|v(x)|)}{\mathrm{t}}}
\end{aligned}
$$

and so (4.2) holds. Therefore, $\left(X, M, *_{P}\right)$ is a fuzzy metric-like space and it is easy to see that $M\left(X, M, *_{p}\right)$ is complete.

Let $S(u(x))=\int_{0}^{L} K(x, r, u(r)) d r$ for all $u \in X$ and $x \in[0, L]$. Obviously, the existence of a solution to the equation (4.1) is equivalent to the existence of a fixed point of $S$.

Theorem 4.1. Suppose that the following hypotheses hold.

(a) $\mathrm{K}:[0, \mathrm{~L}] \times[0, \mathrm{~L}] \times \mathbb{R} \rightarrow \mathbb{R}$ is continuous;

(b) for all $x, r \in[0, \mathrm{~L}]$, there exist a continuous function $\mathrm{h}:[0, \mathrm{~L}] \times[0, \mathrm{~L}] \rightarrow \mathbb{R}$ and $\alpha \in(0,1)$ such that

$$
|\mathrm{K}(\mathrm{x}, \mathrm{r}, \mathrm{u}(\mathrm{r}))|+|\mathrm{K}(\mathrm{x}, \mathrm{r}, \mathrm{v}(\mathrm{r}))|<\alpha \mathrm{h}(\mathrm{x}, \mathrm{r})(|\mathrm{u}(\mathrm{r})|+|v(\mathrm{r})|)
$$

and

$$
\sup _{x \in[0, L]} \int_{0}^{L} h(x, r) d r \leqslant 1 .
$$

Then the integral equation (4.1) has a unique solution $u \in X$. 
Proof. By (4.3) and (4.4), it follows that, for all $x \in[0, \mathrm{~L}]$,

$$
\begin{aligned}
& |S(u(x))|+|S(v(x))|=\left|\int_{0}^{L} K(x, r, u(r)) d r\right|+\left|\int_{0}^{L} K(x, r, v(r)) d r\right| \\
& \leqslant \int_{0}^{\mathrm{L}}|\mathrm{K}(x, r, u(r))| d r+\int_{0}^{\mathrm{L}}|\mathrm{K}(x, r, v(r))| \mathrm{dr} \\
& =\int_{0}^{\mathrm{L}}(|\mathrm{K}(x, \mathrm{r}, \mathrm{u}(\mathrm{r}))|+|\mathrm{K}(x, \mathrm{r}, v(\mathrm{r}))|) \mathrm{d} \mathrm{r} \\
& \leqslant \int_{0}^{L} \alpha h(x, r)(|u(r)+| v(r) \mid) d r \\
& \leqslant \int_{0}^{\mathrm{L}} \alpha \mathrm{h}(x, r) \max _{x \in[0, \mathrm{~L}]}(|\mathrm{u}(x)|+|v(x)|) \mathrm{dr} \\
& =\max _{x \in[0, \mathrm{~L}]}(|\mathrm{u}(\mathrm{x})|+|v(x)|) \int_{0}^{\mathrm{L}} \alpha \mathrm{h}(\mathrm{x}, \mathrm{r}) \mathrm{dr} \\
& \leqslant \alpha \max _{x \in[0, \mathrm{~L}]}(|\mathrm{u}(x)|+|v(x)|),
\end{aligned}
$$

which implies

$$
\max _{x \in[0, \mathrm{~L}]}|\mathrm{S}(\mathrm{u}(x))|+|\mathrm{S}(v(x))| \leqslant \alpha \max _{x \in[0, \mathrm{~L}]}(|\mathfrak{u}(x)|+|v(x)|) .
$$

It follows that

$$
M(S u, S v, t)=e^{-\frac{\max _{x \in[0, L]}|S(u(x))|+|S(v(x))|}{t}} \geqslant(M(u, v, t))^{\alpha}=e^{-\frac{\alpha \max _{x \in[0, L]}(|u(x)|+|v(x)|)}{t}}
$$

for all $t>0$. Let $\varphi(t)=-\ln t$ for all $t \in(0,1]$. Then it follows from (4.5) that

$$
\begin{aligned}
\varphi(M(S u, S v, t)) & =-\ln e^{-\frac{\max _{x \in[0, \mathrm{~L}]}|S(u(x))|+|S(v(x))|}{t}} \\
& =\frac{\max _{x \in[0, \mathrm{~L}]}|S(u(x))|+|S(v(x))|}{t} \\
& \leqslant \alpha \frac{\max _{x \in[0, \mathrm{~L}]}(|\mathrm{u}(\mathrm{x})|+|v(x)|}{\mathrm{t}} \\
& =\alpha\left(-\ln e^{-\frac{\max _{\mathrm{x} \in[0, \mathrm{~L}]}(|\mathrm{u}(\mathrm{x})|+|v(x)|)}{\mathrm{t}}}\right) \\
& =\alpha \varphi(\mathrm{M}(\mathrm{u}, v, \mathrm{t}))
\end{aligned}
$$

for all $t>0$. Thus, from Theorem 3.3, it follows that $S$ has a unique solution in $X$. This completes the proof.

\section{Acknowledgment}

This work is supported by the Fundamental Research Funds for the Central Universities (Grant Number: 2014ZD44) and the Thailand Research Fund under the project RTA5780007. The third and fourth authors were supported by the Royal Golden Jubilee (RGJ) Ph.D. Programme with contract no. PHD/0093/2558 and Chiang Mai University, respectively. The fifth author was supported by Basic Science Research Program through the National Research Foundation of Korea (NRF) funded by the Ministry of Science, ICT and future Planning (2014R1A2A2A01002100).

\section{References}

[1] H. Aydi, A. Felhi, S. Sahmim, Fixed points of multivalued nonself almost contractions in metric-like spaces, Math. Sci. (Springer), 9 (2015), 103-108. 1

[2] H. Aydi, E. Karapınar, Fixed point results for generalized $\alpha-\psi$-contractions in metric-like spaces and applications, Electron. J. Differential Equations, 2015 (2015), 15 pages. 1 
[3] Y. J. Cho, M. Grabiec, V. Radu, On nonsymmetric topological and probabilistic structures, Nova Science Publishers, Inc., New York, (2006). 1

[4] Z. Deng, Fuzzy pseudo-metric spaces, J. Math. Anal. Appl., 86 (1982), 74-95. 1

[5] M. A. Erceg, Metric spaces in fuzzy set theory, J. Math. Anal. Appl., 69 (1979), 205-230. 1

[6] J.-X. Fang, On fixed point theorems in fuzzy metric spaces, Fuzzy Sets and Systems, 46 (1992), 107-113. 1

[7] A. George, P. Veeramani, On some results in fuzzy metric spaces, Fuzzy Sets and Systems, 64 (1994), 395-399. 1

[8] M. Grabiec, Fixed points in fuzzy metric spaces, Fuzzy Sets and Systems, 27 (1988), 385-389. 1, 2

[9] V. Gregori, A. Sapena, On fixed-point theorems in fuzzy metric spaces, Fuzzy Sets and Systems, 125 (2002), 245-252. 1

[10] V. I. Istrăţescu, An introduction to theory of probabilistic metric spaces with applications, (Romanian) Ed. Tehnică Bucureşti, (1974). 2

[11] O. Kaleva, S. Seikkala, On fuzzy metric spaces, Fuzzy Sets and Systems, 12 (1984), 215-229. 1

[12] O. Kramosil, J. Michálek, Fuzzy metrics and statistical metric spaces, Kybernetika (Prague), 11 (1975), 336-344. 1, 2.2

[13] D. Miheț, A Banach contraction theorem in fuzzy metric spaces, Fuzzy Sets and Systems, 144 (2004), 431-439. 1

[14] D. Miheț, On fuzzy contractive mappings in fuzzy metric spaces, Fuzzy Sets and Systems, 158 (2007), $915-921$.

[15] D. Miheț, A class of contractions in fuzzy metric spaces, Fuzzy Sets and Systems, 161 (2010), 1131-1137.

[16] S. N. Mishra, N. Sharma, S. L. Singh, Common fixed points of maps on fuzzy metric spaces, Internat. J. Math. Math. Sci., 17 (1994), 253-258. 1

[17] A. F. Roldán López de Hierro, M. de la Sen, Some fixed point theorems in Menger probabilistic metric-like spaces, Fixed Point Theory Appl., 2015 (2015), 16 pages. 1, 2.7

[18] S. Sharma, Common fixed point theorems in fuzzy metric spaces, Fuzzy Sets and Systems, 127 (2002), 345-352. 1

[19] Y.-H. Shen, D. Qiu, W. Chen, Fixed point theorems in fuzzy metric spaces, Appl. Math. Lett., 25 (2012), 138-141. 3.4

[20] B. Singh, M. S. Chauhan, Common fixed points of compatible maps in fuzzy metric spaces, Fuzzy Sets and Systems, 115 (2000), 471-475. 1

[21] R. Vasuki, A common fixed point theorem in a fuzzy metric space, Fuzzy Sets and Systems, 97 (1998), 395-397. 1 